\title{
What is the relationship of stress to patients' kidney stone-related quality of life?
}

Colin Lundeen ${ }^{1}$; Jonathan R.Z. Lim ${ }^{1}$; Kymora B. Scotland ${ }^{1}$; Reza Safaee Ardekani ${ }^{1}$; Kristina L. Penniston $^{2}$; Necole M. Streeper ${ }^{3}$; Thomas Chi ${ }^{4}$; Jaime Landman ${ }^{5}$; Davis P. Viprakasit ${ }^{6}$; Ben H. Chew ${ }^{1}$

${ }^{1}$ Department of Urologic Sciences, University of British Columbia, Vancouver, BC, Canada; ${ }^{2}$ Department of Urology, University of Wisconsin-Madison, Madison, WI, United States; ${ }^{3}$ Penn State Health, Hershey, PA, United States; ${ }^{4}$ Department of Urology, University of California San Francisco, San Francisco, CA, United States; ${ }^{5}$ Department of Urology, University of California Irvine, Irvine, CA, United States; ${ }^{6}$ Department of Urology, University of North Carolina, Chapel Hill, NC, United States

Cite as: Lundeen C, Lim JRZ, Scotland KB, et al. What is the relationship of stress to patients' kidney stone-related quality of life? Can Urol Assoc J 2020 October 27; Epub ahead of print. http://dx.doi.org/10.5489/cuaj.6652

Published online October 27, 2020

$* * *$

\section{Abstract}

Introduction: Health-related quality of life (HRQOL) is decreased in patients with kidney stones at all stages of stone disease, even when asymptomatic. Stress is thought to contribute to HRQOL, although there has been minimal investigation into the effect of stress on stone-related quality of life (QOL). We used the Wisconsin Stone Quality of Life Questionnaire (WISQOL) to assess the relationship of stress to stone-related QOL in kidney stone patients.

Methods: As part of the WISQOL Research Consortium, patients were approached in outpatient clinics and completed the WISQOL and the Perceived Stress Scale 10-item questionnaire (PSS-

10). Patients with stones at enrollment were divided into those with symptoms and those without, while patients with no current stones formed another group. Questionnaire scores from each group were compared statistically and correlations between the groups were calculated.

Results: Patients ( $n=704)$ were enrolled from six centers. The WISQOL successfully discriminated between patients with current stones and those without $(p<0.0001)$, while the PSS10 did not ( $\mathrm{p}=0.0869)$. The PSS-10 revealed patients with symptomatic kidney stones experienced higher levels of general stress than asymptomatic subjects $(p<0.0001)$. However, a Pearson correlation test comparing the responses from each instrument revealed no overall correlation between general stress and HRQOL $(r=0.05)$.

Conclusions: Symptomatic kidney stones increase general stress, as these patients demonstrate higher PSS-10 scores. Despite this, no correlation exists between general stress and stone-related 
QOL in patients with stone disease regardless of their stone and symptom status. This implies the existence of other factors impacting QOL in these patients, which warrants further exploration.

\section{Introduction}

Kidney stone disease affects nearly 1 in 11 people in the US ${ }^{1}$ with the prevalence increasing over time. ${ }^{2}$ Stone episodes, with resulting medical or surgical management, impact a patient's physical, emotional and psychological well-being. Despite treatment, stone recurrence occurs in over $30 \%$ of patients ${ }^{3}$ which creates a significant burden on patients, their families and the healthcare system. The estimated costs associated with urolithiasis were over $\$ 5.3$ billion in 2005 and are expected to rise. ${ }^{4}$

Anxiety and depression ${ }^{5}$ along with poor health related quality of life (HRQOL) ${ }^{6-13}$ have been associated with urolithiasis. In addition, several studies have identified a correlation between stress and kidney stone disease ${ }^{14-18}$ but no study has found a causative relationship between stress and stone formation.

The Wisconsin stone quality of life questionnaire (WISQOL) is a stone-specific HRQOL tool introduced in 2013 by Penniston and Nakada ${ }^{11}$ and validated in $2017^{21}$ in a diverse population across 8 North American sites. With a goal to accurately characterize stone specific decrements in HRQOL, WISQOL outperformed the validated, 36 item, Short Form General Health Survey (SF-36) in separating out patients with stones and symptoms from those without. The WISQOL questionnaire consists of 28 questions divided into four domains: 1 - social, 2 emotional, 3 - stone related symptom impact and 4 - vitality. The Perceived Stress Scale (PSS10 ) is a validated, widely utilized psychological tool to assess a person's perception of stress. ${ }^{22}$ It is designed to measure general stress with easy to understand questions and has been widely used throughout a variety of medical fields.

The aim of our current multicenter study is to determine if a correlation between stress and HRQOL exists in kidney stone formers.

\section{Methods}

\section{Study design}

Data for this prospective observational study were collected from 6 sites (University of British Columbia, University of California San Francisco, Penn State University, University of California Irvine, University of Wisconsin-Madison and University of North Carolina) within the North American Stone Quality of Life Research Consortium. All sites obtained Institutional Review Board Ethics approval and the University of Wisconsin-Madison was the principal data coordination site. 


\section{Site selection, subjects, and recruitment}

Participants with a history of kidney stones were approached in outpatient clinics at each site to fill out the WISQOL along with the PSS-10 at their initial visit. Eligibility criteria included a history of kidney stones, being 18 years of age or older and the ability to read English. Patients were recruited between June 2016 and April 2018 with approximately 80\% participation. The questionnaires required, on average, 15 minutes to complete.

Patients were divided into three groups. Group 1 included those with a known kidney stone at the initial visit but without any current stone related symptoms. Patients in group 2 also had a recognized stone but were experiencing stone related symptoms. Group 3 consisted of patients with no known stone at the initial visit.

The principal investigator at each site was a urologist, providing medical and surgical stone management per local standard of care. Participation in this study did not alter clinical care in any way.

\section{Data collection}

Consent was obtained for each participant by a member of the study team at each site. The WISQOL, a 28-item, single page, double-sided questionnaire and the PSS-10, a single page questionnaire, were administered upon enrollment to each participant. Demographic information was collected along with medical and surgical data.

\section{Statistical analysis}

The primary study endpoints measured were subject scores on the WISQOL and PSS-10. Their correlation was assessed utilizing Pearson correlation coefficients. Patient demographics and baseline characteristics are summarized descriptively. Data was analyzed using Microsoft ${ }^{\circledR}$ Excel ${ }^{\circledR}$ and IBM ${ }^{\circledR}$ SPSS $^{\mathrm{TM}}$ Statistics, version 23. Statistical significance was determined a priori at $\mathrm{p}<0.05$.

\section{Results}

Seven hundred and four (704) participants were included from 6 centers including 359 women and 345 men. The mean age was $53.1 \pm 15.2$ years. Patients were recruited primarily from a urology or multidisciplinary clinic with $70 \%$ and $29 \%$ from each, respectively. The average BMI was $29.7 \mathrm{~kg} / \mathrm{m}^{2}$ with $69 \%$ of participants being overweight or obese. The majority of participants were Caucasian ethnicity while Latino/Hispanic and Asian populations comprised 8\% and 7\%, respectively. Three hundred and twelve patients had no stone at the time of initial visit while 196 and 181 patients had a stone with and without symptoms, respectively. There were 15 patients with incomplete data who were not included in the comparisons.

When comparing participants with current stones present at the time of study enrollment to those without, the groups were similar but there were statistically significant differences in age at first stone episode, duration of stone history and number of stone related events $(\mathrm{p}<0.05)$ (TABLE 1). In patients with stones and symptoms compared to those with asymptomatic stones 
differences were seen in age, BMI, age at first stone episode and number of prescriptions $(\mathrm{p}<0.05)$ (TABLE 2).

The WISQOL questionnaire identified lower quality of life in participants with stones compared to those without (FIGURE 1). These differences were observed in each of the four WISQOL domains in addition to the total score. Decreased scores for participants with stones compared to those without were seen in each of the 28 individual items compared.

Significant differences were also noted between participants with stones and symptoms and those with asymptomatic stones. (FIGURE 2) Comparing these two categories of participants, the WISQOL revealed higher quality of life for those with no symptoms, even though they had stones present (difference in total score was greater than $30 \%$ ).

Unlike the WISQOL, the PSS-10 failed to show a difference in general stress between participants with and without stones. (FIGURE 3) On the other hand, the PSS-10 distinguished participants with symptomatic stones from those with stones but no symptoms. Participants with stones and symptoms had higher general stress levels than those with stones but no symptoms. In similar fashion the PSS-10 differentiated between patients with symptomatic stones and those with no stones $(\mathrm{p}<0.001)$.

When comparing the two questionnaires with Pearson's correlation coefficients, no correlation was seen between participants with stones and those without both in total scores and in each individual domain. (TABLE 3) Despite both questionnaires identifying differences in participants with stones and symptoms from those without, there was no correlation between WISQOL and PSS-10 for these groups.

\section{Discussion}

This multicenter study shows that stress and HRQOL appear to be unrelated in kidney stone patients. While a possible relationship has been suggested in prior studies, our results indicate that general stress is not a driver of HRQOL in stone formers. A physiological link between stress and stones was posited by Walters in $1986 .{ }^{19}$ His theory proposed that the stress response led to increased vasopressin levels, retention of free water and hypertonic urine. In addition, ACTH release in response to stress results in stimulation of the parathyroid glands and increased serum calcium.

Since that time, many other authors have investigated the link between stress and kidney stone formation. Diniz et al. found that patients presenting with an acute episode of renal colic had a nearly threefold risk of having undergone significantly stressful life events than controls. ${ }^{15}$ Looking more closely at blood and urinary components of stone formation in relation to stress and HRQOL Arzoz-Fabregas et al. found that chronic stress affects stone formers and may contribute to recurrent calcium oxalate stones. ${ }^{17}$ However, in 2017 the same authors re-evaluated this data, using urinary marker ratios and quotients, and found no significant differences between the groups related to urinary risk factors..$^{20}$ 
In recent years, medical research has placed an emphasis on investigating HRQOL. The relationship between stones and quality of life factors such as stress and depression have been studied. Early data were published on the impact of stress and kidney stone formation in 1997 by Najem et al. ${ }^{14}$ They found, in a case control study, that multiple stress factors were higher in stone formers compared to controls. The most significant of these factors were low family income, stressful mortgage problems and emotional life issues. Angell et al. reported an increased risk of psychological distress in their cohort of stone formers compared to the general population. ${ }^{5}$ Over $30 \%$ of their cohort reported significant psychological distress as opposed to only $16 \%$ of Americans in general. When assessing overall HRQOL in stone formers the SF-36 was utilized in early stone studies. Diniz ${ }^{7}$, Penniston ${ }^{6}$ and Bensalah ${ }^{8}$ and their groups all found decreased HRQOL in stone formers compared to controls.

In 2013 Penniston and Nakada developed the Wisconsin Stone Quality of Life Questionnaire (WISQOL), a novel tool to assess disease-specific HRQOL in kidney stone patients. ${ }^{11}$ The WISQOL was then validated in a multi-national prospective study and delineated HRQOL decrements in patients with stones from those without. ${ }^{21}$ The questionnaire was more sensitive than the SF-36 at detecting HRQOL changes. In addition, it detected HRQOL differences in patients with symptomatic stones from those with stones but no symptoms. In our study the WISQOL performed well at identifying HRQOL differences in participants with stones from those without along with those with stones and symptoms from those with stones but no symptoms. This further supports the use of the WISQOL in HRQOL research for kidney stone formers.

We hypothesized that stress would be negatively correlated with HRQOL. Using the WISQOL and PSS-10 tools, we discovered that no clear relationship exists between these two entities for kidney stone formers. While the PSS-10 discriminated between participants with symptomatic stones from those with asymptomatic stones, it was unable to differentiate participants with stones from those without. This was due to patients with stones and no symptoms having equivalent PSS-10 scores to patients without stones. Similar findings were seen in a study by Miyaoka et al. where symptomatic stones and frequent passage of stones were related to increased perceived stress using the PSS-10. ${ }^{16}$ In that study it was noted that non-stone related characteristics such as female sex, employment status and recent traumatic events also impacted PSS-10 scores. Our findings expand on the suggestion that stone symptoms, as opposed to stone disease itself, cause an elevation in general stress. The PSS-10 appears to be capturing general stress as opposed to stress specifically related to stones. In contrast, the WISQOL filters out general stress and focuses in on HRQOL in stone disease.

We believe that this is the first study looking directly at the relationship between stress and HRQOL using validated questionnaires. Our hypothesis that increased stress leads to lower quality of life for stone formers was disproven. There does not appear to be a direct link between general stress and HRQOL suggesting that the PSS-10 measures stress unrelated to stone disease. 
The study has a few limitations. First, the WISQOL is available only in English which excludes a portion of the population and reduces generalizability. Second, the study was performed only at academic centers and may not be applicable to the private practice or community setting. Third, most data were captured at outpatient appointments so may miss a relationship between stress and HRQOL that exists during an acute stone episode. Finally, this study looked at a single time point and may miss changes in stress and HRQOL over time.

\section{Conclusions}

General stress does not appear to be related to quality of life for kidney stone patients. Higher levels of stress in symptomatic patients, though, suggests that symptoms may add to stress levels. The WISQOL is more sensitive than the PSS -10 at identifying the adverse psychological effects of kidney stone disease. While the WISQOL revealed decrements in quality of life for patients with stones compared to those without, the PSS-10 was unable to differentiate between these groups. These findings would suggest that factors other than stress drive changes in HRQOL for stone formers.

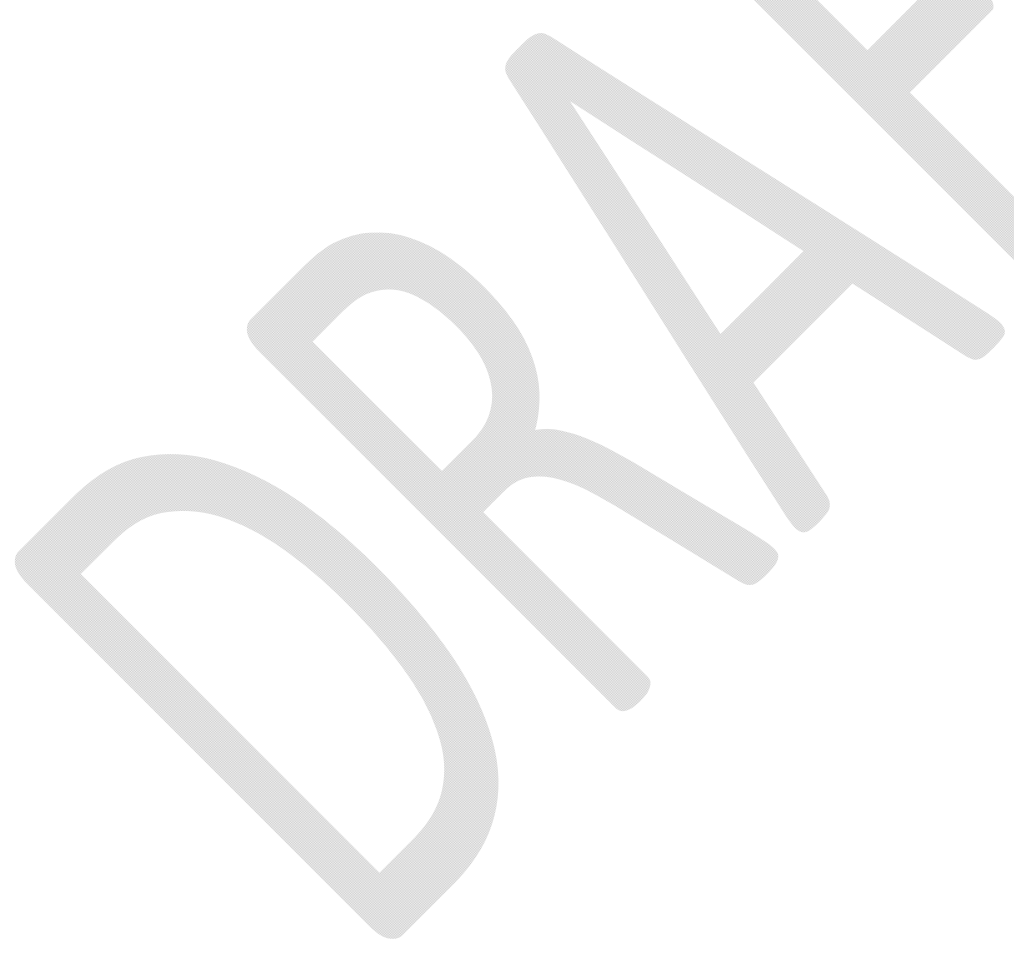




\section{References}

1. Scales CD, Smith AC, Hanley JM, Saigal CS, Project UDiA. Prevalence of kidney stones in the United States. Eur Urol 2012;62:160-65.

2. Stamatelou KK, Francis ME, Jones CA, Nyberg LM, Curhan GC. Time trends in reported prevalence of kidney stones in the United States: 1976-1994. Kidney Int 2003;63:181723.

3. Rule AD, Lieske JC, Li X, Melton LJ, Krambeck AE, Bergstralh EJ. The ROKS nomogram for predicting a second symptomatic stone episode. J Am Soc Nephrol 2014;25:2878-86.

4. Pearle MS, Calhoun EA, Curhan GC, Project UDoA. Urologic diseases in America project: urolithiasis. J Urol 2005;173:848-57.

5. Angell J, Bryant M, Tu H, Goodman M, Pattaras J, Ogan K. Association of depression and urolithiasis. Urology 2012;79:518-25.

6. Penniston KL, Nakada SY. Health related quality of life differs between male and female stone formers. J Urol 2007;178:2435-40; discussion 2440.

7. Diniz DH, Blay SL, Schor N. Quality of life of patients with nephrolithiasis and recurrent painful renal colic. Nephron Clin Pract 2007;106:c91-7.

8. Bensalah K, Tuncel A, Gupta A, Raman JD, Pearle MS, Lotan Y. Determinants of quality of life for patients with kidney stones. J Urol 2008;179:2238-43; discussion 2243.

9. Donnally CJ, Gupta A, Bensalah K, et al. Longitudinal evaluation of the SF-36 quality of life questionnaire in patients with kidney stones. Urol Res 2011;39:141-46.

10. Bryant M, Angell J, Tu H, Goodman M, Pattaras J, Ogan K. Health related quality of life for stone formers. J Urol 2012;188:436-40.

11. Penniston KL, Nakada SY. Development of an instrument to assess the health related quality of life of kidney stone formers. J Urol 2013;189:921-30.

12. Modersitzki F, Pizzi L, Grasso M, Goldfarb DS. Health-related quality of life (HRQoL) in cystine compared with non-cystine stone formers. Urolithiasis 2014;42:53-60.

13. Patel N, Brown RD, Sarkissian C, De S, Monga M. Quality of life and urolithiasis: the patient - reported outcomes measurement information system (PROMIS). Int Braz J Urol 2017;43:880-86.

14. Najem GR, Seebode JJ, Samady AJ, Feuerman M, Friedman L. Stressful life events and risk of symptomatic kidney stones. Int J Epidemiol 1997;26:1017-23.

15. Diniz DH, Schor N, Blay SL. Stressful life events and painful recurrent colic of renal lithiasis. J Urol 2006;176:2483-87; discussion 2487.

16. Miyaoka R, Ortiz-Alvarado O, Kriedberg C, Alanee S, Chotikawanich E, Monga M. Correlation between stress and kidney stone disease. J Endourol 2012;26:551-55.

17. Arzoz-Fàbregas M, Ibarz-Servio L, Fernández-Castro J, et al. Chronic stress and calcium oxalate stone disease: influence on blood cortisol and urine composition. Urology 2013;82:1246-52.

18. Arzoz-Fabregas M, Ibarz-Servio L, Edo-Izquierdo S, Doladé-Botías M, Fernandez-Castro J, Roca-Antonio J. Chronic stress and calcium oxalate stone disease: is it a potential recurrence risk factor? Urolithiasis 2013;41:119-27. 
19. Walters DC. Stress as a principal cause of calcium oxalate urolithiasis. Int Urol Nephrol. 1986;18:271-75.

20. Arzoz-Fabregas M, Roca-Antonio J, Ibarz-Servio L, Jappie-Mahomed D, Rodgers A. Stress-stones-stress-recurrent stones: a self-propagating cycle? Difficulties in solving this dichotomy. Urolithiasis 2017;45:515-24.

21. Penniston KL, Antonelli JA, Viprakasit DP, et al. Validation and Reliability of the Wisconsin Stone Quality of Life Questionnaire. J Urol 2017;197:1280-88.

22. Cohen S, Kamarck T, Mermelstein R. A global measure of perceived stress. J Health Soc Behav 1983;24:385-96. 


\section{Figures and Tables}

Fig. 1. Comparison of Wisconsin Stone Quality of Life (WISQOL) scores between patients who reported having stones vs. those reporting no stones at the time they completed the WISQOL and the Perceived Stress Scale (PSS-10) questionnaire

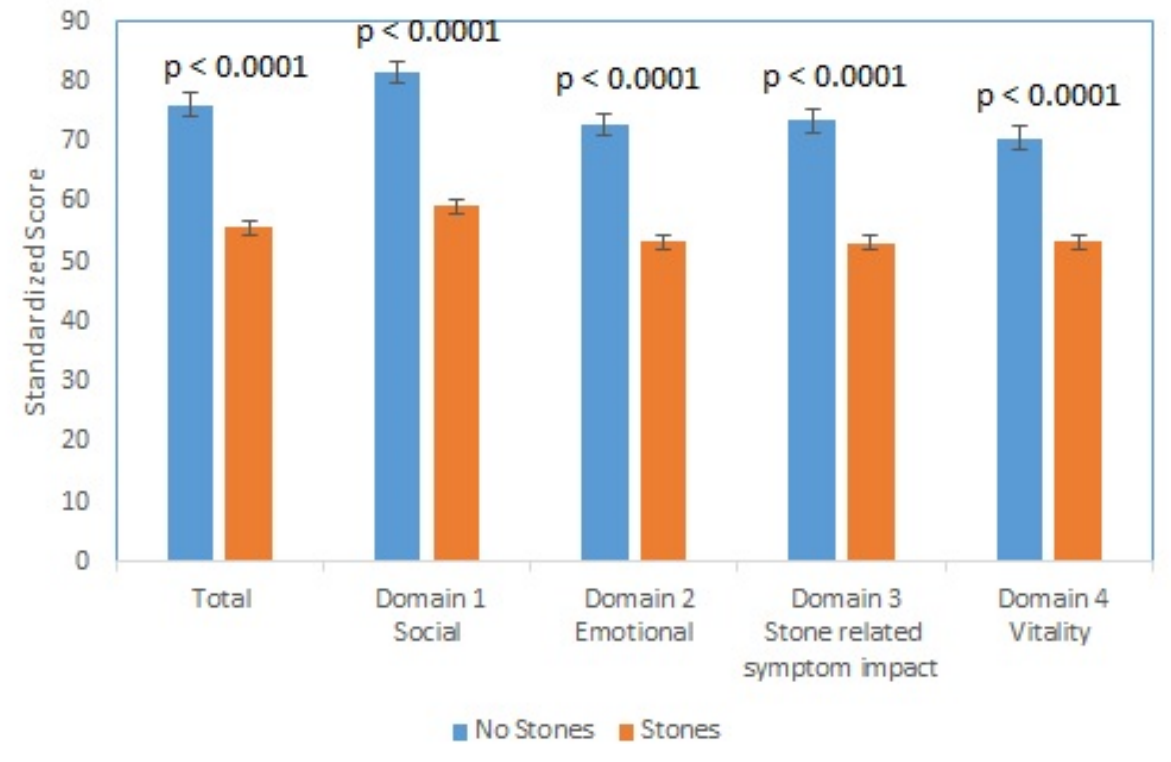

Fig. 2. Comparison of Wisconsin Stone Quality of Life (WISQOL) questionnaire scores between patients who reported having stones and stone-related symptoms vs. those reporting stones but no symptoms at the time they completed the WISQOL and the Perceived Stress Scale (PSS-10).

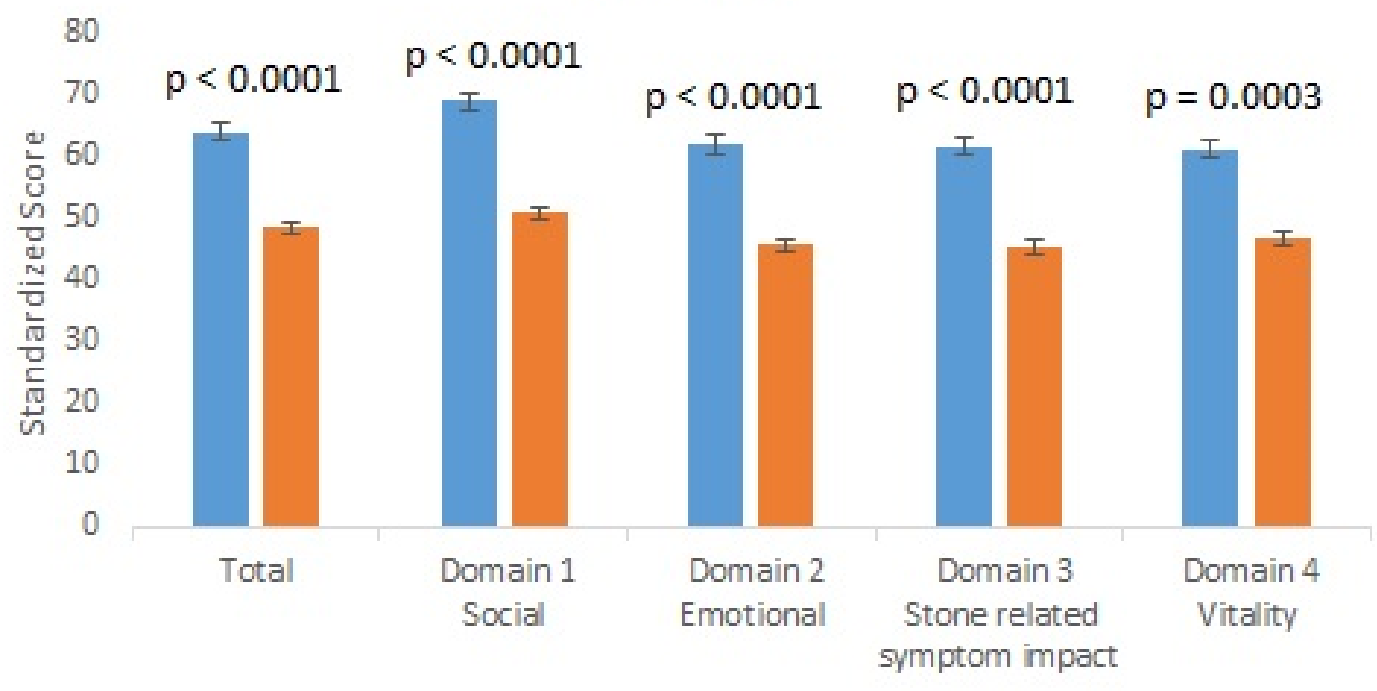

- Stones no symptoms a Stones and symptoms 
Fig. 3. Comparison of Perceived Stress Scale (PSS-10) scores between groups.

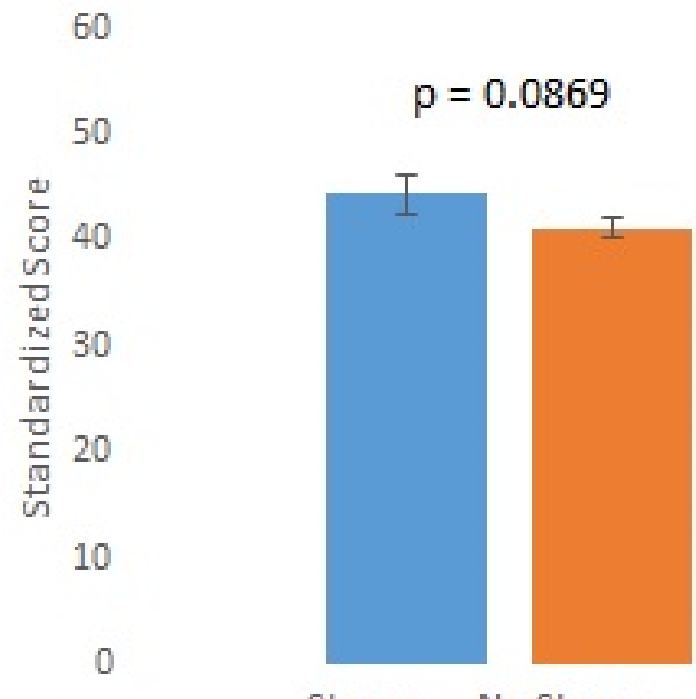

Stones No Stones

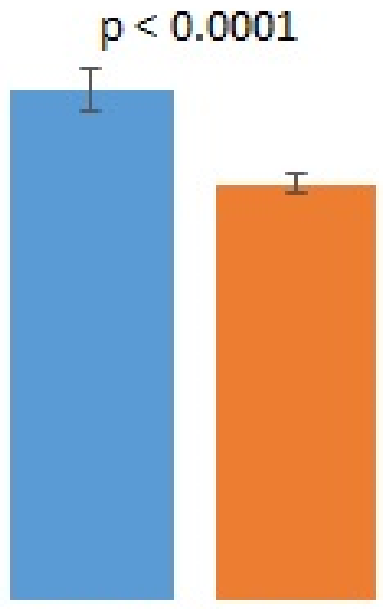

Stones/symptoms Stones no symptoms

\begin{tabular}{|l|c|c|c|}
\hline \multicolumn{4}{|l|}{$\begin{array}{l}\text { Table 1. Baseline characteristics of patients who reported having stones vs. those } \\
\text { reporting no stones at the time they completed the WISQOL and the PSS-10 }\end{array}$} \\
\hline & Stones & No stones & p \\
\hline Age (yrs) & $52.2 \pm 15.0$ & $54.5 \pm 15.4$ & 0.091 \\
\hline BMI (kg/m ${ }^{2}$ & $29.7 \pm 7.8$ & $29.8 \pm 7.6$ & 0.852 \\
\hline First stone episode (age in yrs) & $39.7 \pm 16.6$ & $44.4 \pm 17.2$ & 0.003 \\
\hline Duration of stone disease (yrs) & $13.1 \pm 13.6$ & $10.5 \pm 12.6$ & 0.040 \\
\hline Estimated number of events & $8.9 \pm 16.6$ & $4.8 \pm 10.8$ & 0.005 \\
\hline Events per year & $2.4 \pm 4.5$ & $1.9 \pm 3.2$ & 0.197 \\
\hline Number of comorbidities & $1.8 \pm 1.8$ & $2.0 \pm 1.9$ & 0.108 \\
\hline Number of prescriptions & $1.5 \pm 1.6$ & $1.5 \pm 1.5$ & 0.951 \\
\hline Percentage on diuretic therapy & 14 & 15 & 0.739 \\
\hline Percentage on alkali therapy & 17 & 20 & 0.367 \\
\hline
\end{tabular}

Reported as mean \pm standard deviation. BMI: body mass index; PSS-10: Perceived Stress Scale; WISQOL: Wisconsin Stone Quality of Life. 
Table 2. Baseline characteristics of patients who reported having stones and stonerelated symptoms vs. those reporting stones but no symptoms at the time they completed the WISQOL and the PSS-10

\begin{tabular}{|l|c|c|c|}
\hline & Stones + symptoms & $\begin{array}{l}\text { Stones with no } \\
\text { symptoms }\end{array}$ & p \\
\hline Age (yrs) & $48.9 \pm 13.8$ & $55.8 \pm 16.0$ & $<0.001$ \\
\hline BMI $\left(\mathrm{kg} / \mathrm{m}^{2)}\right.$ & $30.4 \pm 8.4$ & $28.7 \pm 7.0$ & 0.047 \\
\hline First stone episode (age in yrs) & $37.4 \pm 15.2$ & $42.1 \pm 14.25$ & 0.011 \\
\hline Duration of stone disease (yrs) & $11.7 \pm 11.9$ & $14.3 \pm 14.6$ & 0.085 \\
\hline Estimated number of events & $10.3 \pm 18.9$ & $7.0 \pm 12.0$ & 0.071 \\
\hline Events per year & $2.6 \pm 5.3$ & $2.3 \pm 3.6$ & 0.496 \\
\hline Number of comorbidities & $1.6 \pm 1.7$ & $2.0 \pm 1.8$ & 0.051 \\
\hline Number of prescriptions & $1.3 \pm 1.5$ & $1.7 \pm 1.6$ & 0.013 \\
\hline Percentage on diuretic therapy & 12 & 14 & 0.276 \\
\hline Percentage on alkali therapy & 16 & 20 & 0.139 \\
\hline
\end{tabular}

Reported as mean \pm standard deviation. BMI: body mass index; PSS-10: Perceived Stress Scale; WISQOL: Wisconsin Stone Quality of Life.

\begin{tabular}{|l|c|c|}
\hline \multicolumn{3}{|l|}{ Table 3. Correlation between WISQOL and PSS-10 } \\
\hline $\begin{array}{l}\text { Correlation between WISQOL and PSS- } \\
\mathbf{1 0}\end{array}$ & $\begin{array}{c}\text { Pearson's } \\
\text { correlation } \\
\text { coefficient (r) }\end{array}$ & $\mathbf{p}$ \\
\hline Total & -0.05 & 0.621 \\
\hline Domain 1 (Social) & -0.001 & 0.571 \\
\hline Domain 2 (Emotional) & -0.061 & 0.605 \\
\hline Domain 3 (Stone-related symptom impact) & -0.074 & 0.188 \\
\hline Domain 4 (Vitality) & -0.121 & 0.050 \\
\hline
\end{tabular}

PSS-10: Perceived Stress Scale; WISQOL: Wisconsin Stone Quality of Life. 